\title{
Correspondence
}

\section{BAG3 is required for IKK $\alpha$ nuclear translocation and emergence of castration resistant prostate cancer}

\author{
M Ammirante ${ }^{1}$, V De Laurenzi ${ }^{2,3}$, V Graziano ${ }^{2}$, MC Turco $^{3,4}$ and A Rosati ${ }^{\star 3,4}$ \\ Cell Death and Disease (2011) 2, e139; doi:10.1038/cddis.2011.23; published online 31 March 2011 \\ Subject Category: Cancer
}

\section{Dear Editor,}

Prostate cancer $(\mathrm{CaP})$ is a common cause of cancer death in males in Europe and North America. ${ }^{1,2}$ Despite screening for prostate-specific antigen has led to an early diagnosis, it has not resulted in the desired decrease of mortality mainly due to cancer recurrence and metastatic desease. Although radical prostatectomy, radiation and androgen ablation are effective therapies for androgen-dependent $\mathrm{CaP}$, metastatic castration-resistant (CR) $\mathrm{CaP}$ is a major complication associated with high mortality rates. Although most patients initially respond to androgen ablation therapy, many become resistant within 18 months. ${ }^{1,2}$ It is therefore of uttermost importance to determine the molecular mechanism leading to the emergence of $\mathrm{CR}-\mathrm{CaP}$ and to identify predictive markers of progression. Recently, we have shown that castration results in massive inflammatory infiltration in the tumor remnant, and that infiltrating $B$ cells promote tumor growth by lymphotoxin production that results in $\mathrm{IKK} \alpha$ nuclear translocation and activation that in turn promotes tumor growth with a NF- $\kappa \mathrm{B}$-independent mechanism. ${ }^{3}$

We have previously shown that BAG3, a member of the BAG family of heat-shock proteins co-chaperons, is overexpressed in numerous cancers and promotes tumor cell survival $^{4,5}$ by preventing HSP-70-dependent IKK $\gamma$ degrada-
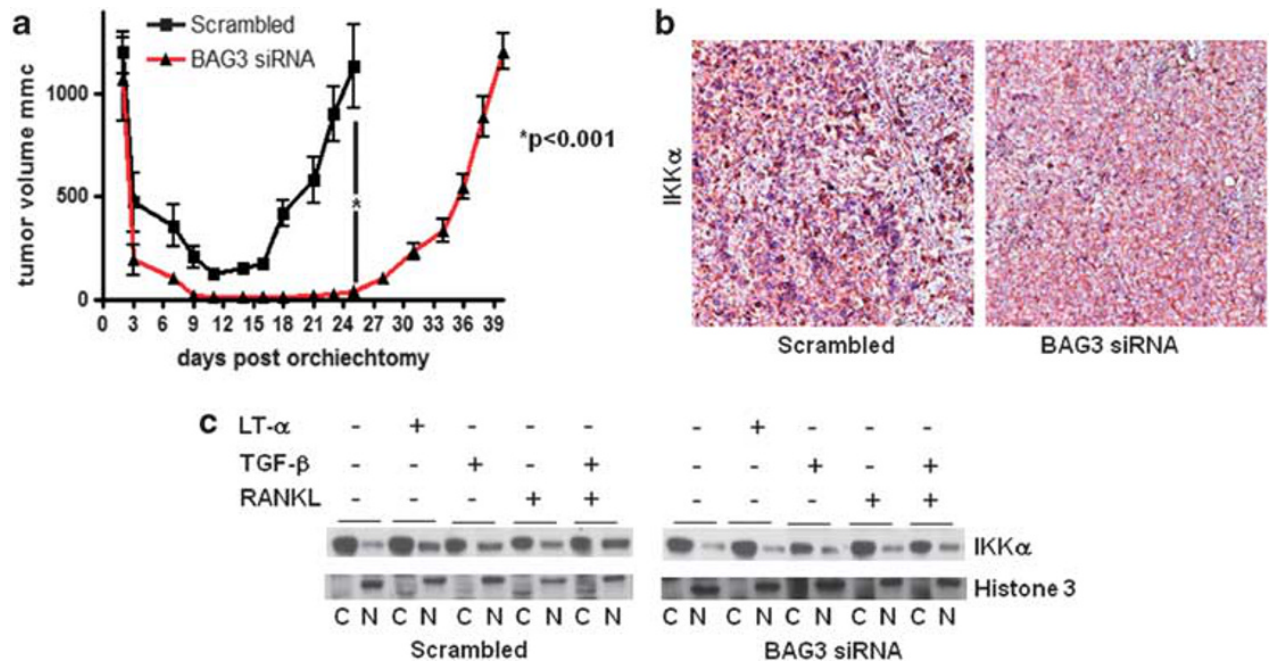

Figure 1 (a) myc-CaP cells derived from the FVB background were provided by C Sawyers (University of California, Los Angeles and Memorial Sloan Kettering Cancer Center). ${ }^{3}$ Virus-containing supernatants were added to the cells for 2 days with polybrene, and transduced cells were selected in $5 \mu \mathrm{g} / \mathrm{ml} \mathrm{puromycin} \mathrm{(Invitrogen,} \mathrm{Carlsbad,} \mathrm{CA,}$ USA). Male FVB mice were injected with myc-CaP cells, previously infected with lentiviruses expressing control (Scrambled) or BAG3-specific siRNAs. When tumors reached $900 \mathrm{~mm}^{3}$, mice ( $n=10$ per group) were castrated. Tumor size was measured every 2-3 days. Results are expressed as means \pm S.D. Data were analyzed by Student's $t$-test using GraphPad Prism statistical program ( ${ }^{*} P<0.001$ ). (b) Paraffin sections of tumors transduced with BAG3 siRNA (right panel) or control vector (left panel) as described in a were stained using a specific antibody recognizing IKK $\alpha$ (Imgenex, San Diego, CA, USA) and counterstained with hematoxylin as previously described ${ }^{3}$ (original magnification $\times 200$ ). (c) myc-CaP cells were infected with a lentivirus carrying a control (Scrambled; left panel) or a BAG3-specific siRNA (right panel) then treated with LT $\alpha 2 \beta 1$, TGF $\beta$ or RANKL, all at $10 \mathrm{ng} / \mathrm{ml}$ (Sigma, Aldrich, St. Louis, MO, USA) for $1 \mathrm{~h}$. Cells were harvested and subjected to nuclei/cytosol fractionation. Lysates were analyzed by immunoblot, using the same antibody as in $\mathbf{b}$ (C, cytosol; N, nucleus).

${ }^{1}$ Laboratory of Gene Regulation and Signal Transduction, Department of Pharmacology and Cancer Center, School of Medicine, University of California, La Jolla, CA, USA; ${ }^{2}$ Department of Biomedical Sciences, University of Chieti-Pescara, Chieti, Italy; ${ }^{3}$ BIOUNIVERSA S.R.L., Fisciano, Italy and ${ }^{4}$ Department of Pharmaceutical and Biomedical Sciences (FARMABIOMED), University of Salerno, Fisciano, Italy

*Corresponding author: A Rosati, Cellular Biochemistry Lab no. 56, Division of Biomedicine 'Arturo Leone', Department of Pharmaceutical and Biomedical Sciences, University of Salerno. Via Ponte Don Melillo, Fisciano 84084 (SA), Italy. Tel: + 3908996 9430; Fax: + 3908996 9602; E-mail: arosati@ unisa.it 
tion, thus sustaining NF- $\mathrm{BB}$ activity. ${ }^{6}$ We and others have shown that BAG3 is expressed in $\mathrm{CaPs}^{7,8}$ we therefore investigated the possibility that BAG3 has a role also in CR-CaP growth. To this end we examined if BAG3 deletion was able to delay the emergence of CR-CaP, using the myc-CaP allograft model previously described. ${ }^{3}$ BAG3 short hairpin RNA (shRNA) was cloned into pLSLPw, and lentivirus stocks prepared as previously described. ${ }^{9}$ myc-CaP cells were transduced with the virus and selected in puromycin. FVB male mice were SC injected with myc-CaP cells, previously infected with lentiviruses expressing control (Scrambled) or BAG3-specific siRNAs. When tumors reached $900 \mathrm{~mm}^{3}$, mice were castrated and tumor size measured every 2-3 days. As shown in Figure 1a, downregulation of BAG3 significantly delays the emergence of CR-CaP tumors following androgen ablation. The delay in tumor growth observed in BAG3-silenced tumors resembles the delay observed when $\mathrm{IKK} \alpha$ is deleted, suggesting that BAG3 modulation was affecting IKK $\alpha$ levels or localization. Interestingly, tumor staining for $\mathrm{IKK} \alpha$ shows that reduction of BAG3 results in a strong inhibition of IKK $\alpha$ nuclear translocation (Figure 1b). We therefore investigated whether BAG3 was required for IKK $\alpha$ nuclear translocation in response to inflammatory cytokines. To this purpose, myc-CaP cells were infected with lentivirus carrying a BAG3-specific shRNA or a scrambled control sequence and treated for $1 \mathrm{~h}$ with $\mathrm{LT}-\alpha$, or TGF- $\beta$ or RANKL. As shown in Figure 1c, treatment with any of this cytokines results in IKK $\alpha$ nuclear translocation that is strongly reduced in cells transduced with BAG3 shRNA. These results demonstrate that BAG3 is required for $\mathrm{IKK} \alpha$ nuclear translocation in response to inflammatory cytokines.

Altogether, our data demonstrate that BAG3 has an essential role in promoting the emergence of CR-CaP by modulating $\mathrm{IKK} \alpha$ nuclear translocation in response to cytokines produced by infiltrating inflammatory cells, most likely B-cell-derived lymphotoxin in accordance with our

previously published data. ${ }^{3}$ These observation suggest that BAG3 is a potential marker of CaP aggressiveness and of response to androgen ablation therapy, and is also a potential target for future therapies. More work needs to be performed to clearly correlate BAG3 expression to clinical outcome as well as to clarify the molecular mechanism through which BAG3 influences IKK $\alpha$ nuclear translocation. We can imagine at least two scenarios, in the first one BAG3 is required to release $\mathrm{IKK} \alpha$ from another binding protein that prevents its nuclear translocation, in the second one BAG3 itself would shuttle IKK $\alpha$ to the nucleus, in support of this second mechanism are some preliminary data from our laboratory showing BAG3 nuclear localization in some $\mathrm{CaP}$ cell lines.

\section{Conflict of interest}

The authors declare no conflict of interest.

Acknowledgements. This work was made possible by a grant awarded from Associazione Italiana per la Ricerca sul Cancro (AIRC) to MA.

\footnotetext{
1. Shepard DR, Raghavan D. Nat Rev Clin Oncol 2010; 7: 13-21.

2. Macfarlane RJ, Chi KN. Urol Clin North Am 2010; 37: 105-119.

3. Ammirante $\mathrm{M}$ et al. Nature 2010; 464: 302-305.

4. Rosati A et al. IJBCB 2007; 39: 1337-1342.

5. Romano MF et al. Cell Death Differ 2003; 10: 383-385.

6. Ammirante $M$ et al. Proc Natl Acad Sci USA 2010; 107: 7497-7502.

7. Iwasaki M et al. BAG3 Cancer Res 2007; 67: 10252-10259.

8. Staibano $S$ et al. Tumour Biol 2010; 31: 461-469.

9. Luo JL et al. Nature 2007; 446: 690-694.
}

(c)

OMERIEHTS RESERVED censed under the Creative Commons Attribution-Noncommercial-No Derivative Works 3.0 Unported License. To view a copy of this license, visit http://creativecommons.org/licenses/by-nc-nd/3.0/ 